\title{
Phenomenology of Infrared Finite Gluon Propagator and Coupling Constant
}

\author{
A. A. Natale \\ Instituto de Física Teórica - UNESP - Rua Pamplona, 145 - 01405-900 - São Paulo - SP - Brazil
}

(Received on September 14, 2006)

\begin{abstract}
We report on some recent solutions of the Dyson-Schwinger equations for the infrared behavior of the gluon propagator and coupling constant, discussing their differences and proposing that these different behaviors can be tested through hadronic phenomenology. We discuss which kind of phenomenological tests can be applied to the gluon propagator and coupling constant, how sensitive they are to the infrared region of momenta and what specific solution is preferred by the experimental data.
\end{abstract}

Keywords: Gluon propagator; Schwinger-dyson equations; Gluon mass

\section{INTRODUCTION}

During the last years there has been much effort in trying to obtain the infrared (IR) behavior of the Quantum Chromodynamics (QCD) Green's functions by means of theoretical and phenomenological studies [1-11] and by simulations of QCD on a lattice [12-15]. The infrared behavior of the gluon and ghost propagators, as well as of the coupling constant are completely intertwined with the confinement problem. For example, if the gluon propagator would be as singular as $1 / k^{4}$ when $k^{2} \rightarrow 0$, it would indicate an interquark potential rising linearly with the separation.

In the late seventies, working in the Landau gauge and Euclidean space, Mandelstam obtained a solution of the Dyson-Schwinger equation (DSE) for the gluon propagator, in the case of pure gauge QCD, that behaved as $1 / k^{4}[16]$. A few years later Cornwall obtained a gauge invariant solution that behaved as $1 /\left[k^{2}+m^{2}\left(k^{2}\right)\right]$ [17]. In this last case, as $k^{2} \rightarrow 0$, the function $m^{2}\left(k^{2}\right)$ was interpreted as a dynamical gluon mass with the limit $m^{2}\left(k^{2} \rightarrow 0\right)=m_{g}^{2}$. Both solutions reproduce the expected perturbative behavior naturally at large $k^{2}$. The $1 / k^{4}$ result was named as a "confining solution", whereas the massive (or any infrared finite solution) became known as a "confined solution". It is clear that for the confined solution a more sophisticated explanation of quark confinement must be at work, and for this reason the appealing $1 / k^{4}$ solution became more popular, but not without some debate [18].

Recently the coupled DSE equations for the gluon and ghost propagators were solved with different approximations [2], resulting in an infrared gluon propagator that behaves roughly as $k^{2} /\left[k^{4}+a^{4}\right]$, i.e. vanishes as $k^{2} \rightarrow 0$. This kind of behavior had also been predicted by Gribov [19] and Zwanziger [20] in a different approach. The behavior of gluon and ghost propagators in Euclidean Yang-Mills theory quantized in the maximal Abelian gauge (MAG) were also studied considering the effects arising from a treatment of Gribov copies in the MAG and those arising from a dynamical mass originating in a dimension two gluon condensate [21]. In these studies the infrared gluon propagator depends on the so called Gribov parameter, whose variation seems to make an interpolation between the vanishing propagator and the massive one!

A great step ahead in this problem has also been provided by the QCD lattice simulations in Landau gauge, which strongly support the existence of an infrared finite gluon propagator [12-15], where by finite we mean that the gluon propagator may be zero or different from zero at $k^{2}=0$. This is interesting enough, because indicates the appearance of a dynamical mass scale for the gluon, which imply in the existence of a non-trivial QCD infrared fixed point, i.e. the freezing of the coupling constant at the origin of momenta [8]. Unfortunately the lattice data is not precise enough to definitively settle the questions of Green's functions at the origin of momenta, but there are claims that the results can nicely accommodate a massive gluon solution like the one proposed by Cornwall [13]. Notice that at first glance it seems that these two possibilities are just a small detail, however they imply in two different confinement scenarios. Cornwall indicated that a dynamically generated gluon mass induces vortex solutions in the theory and these are responsible for the quark confinement [17]. On the other hand the vanishing gluon propagator jointly with an infrared enhanced ghost propagator may induce an effective linear confining potential between quarks [22]. It is not surprising that DSE for the QCD propagators lead to different solutions as long as they are solved with different truncations and approximations, as, for instance, the choice of the trilinear gluon vertex plays a crucial role in the solution. This means that we should wait for still more improved DSE solutions or lattice simulations for the gluon propagator.

It is our purpose here to advocate that the hadronic phenomenology can provide solid information about the infrared behavior for the gluon propagator and coupling constant. Which kind of phenomenological tests are possible to propose in order to study the infrared behavior of the gluon propagator and coupling constant? In our opinion there are two possibilities: (i) We can make use of QCD inspired models where it is assumed "a priori" that the gluon propagator and running coupling constant must have one specific non-perturbative behavior, as, for example, happens in the Pomeron model of Landshoff and Nachtmann [23]; or (ii) we can consider perturbative QCD calculations introducing an effective propagator and coupling constant in the sense of the "Dynamical Perturbation Theory" (DPT) proposed by Pagels and Stokar [24] many 
years ago. In the next section we will detail and discuss examples of these two possibilities.

\section{PHENOMENOLOGICAL TESTS FOR THE INFRARED BEHAVIOR OF THE GLUON PROPAGATOR AND COUPLING CONSTANT}

\section{A. Testing the IR behavior in a QCD Pomeron model}

It is known that a connection between the theoretical predictions of the perturbative Pomeron described by the BFKL Pomeron [25] and the experimental data is far from being fully understood. Landshoff and Nachtmann proposed a simple Pomeron model where the gluon propagation is affected by the non-trivial QCD vacuum leading naturally to a correlation length (or gluon mass scale). The existence of this correlation length is the only feasible explanation to the fact that diffractive interactions mediated by the Pomeron (which is assumed to be composed by at least two gluons in a color singlet state exchanged in channel $t$ between hadrons) obey the additive quark rule. This model is quite suitable to test IR properties of coupling constant and gluon propagator obtained through DSE, because it always involves one integration over the product of gluons propagators and coupling constant $\left(g^{2}\left(k^{2}\right) \times D\left(k^{2}\right)\right)$.

As long as we deal with a QCD inspired model for the Pomeron we should be aware that the model will be valid up to a certain scale or approximation. Comparing the theoretical predictions of the Landshoff and Nachtmann (LN) Pomeron model with the experimental data we can be quite confident that the model is very reasonable to compute diffractive scattering in the small transferred momentum limit (i.e. small $t$ physics). The Pomeron, in the LN model, is described as the singlet channel exchange of two non-perturbative gluons between hadrons, and in the sequence we discuss the case of elastic differential cross section for proton-proton scattering.

In the $\mathrm{LN}$ model the elastic differential cross section can be obtained from

$$
\frac{d \sigma}{d t}=\frac{|A(s, t)|^{2}}{16 \pi s^{2}}
$$

where the amplitude for elastic proton-proton scattering via two-gluon exchange can be written as

$$
A(s, t)=i s 8 \alpha_{s}^{2}\left[T_{1}-T_{2}\right]
$$

with

$$
\begin{gathered}
T_{1}=\int_{0}^{s} d^{2} k D\left(\frac{q}{2}+k\right) D\left(\frac{q}{2}-k\right)\left|G_{p}(q, 0)\right|^{2} \\
T_{2}=\int_{0}^{s} d^{2} k D\left(\frac{q}{2}+k\right) D\left(\frac{q}{2}-k\right) G_{p}\left(q, k-\frac{q}{2}\right) \\
\times\left[2 G_{p}(q, 0)-G_{p}\left(q, k-\frac{q}{2}\right)\right]
\end{gathered}
$$

where $G_{p}(q, k)$ is a convolution of proton wave functions

$$
G_{p}(q, k)=\int d^{2} p d \kappa \psi^{*}(\kappa, p) \psi(\kappa, p-k-\kappa q)
$$

In Eq.(3) $D\left(q^{2}\right)$ is the non-perturbative expression for the gluon propagator. Note that the loop integral in Eq.(3) contains the product of two gluon propagators weighted by $G_{p}(q, k)$, which will be written in terms of proton form factors. This is a general behavior, where the form factors will vary according to the type of hadron involved in the scattering. Evidently this means that the integral will depend on different scales contained in each form factor, which in the particular case of the proton is the proton mass, whereas for pion-proton, $\gamma-\rho$ scattering, or others, we have different mass scales in the integration, in addition to the one that enters in the gluon propagator and coupling constant. Another important feature of this model is the kinematic structure, where one of the gluons carry most of the momentum. The other gluon just seems to enter in the process with a very small momentum in order to form a color singlet, but it is responsible for the most important part of the integration area [26].

To obtain the cross section for the elastic differential $p-p$ scattering we use the gluon propagator in Landau gauge written as

$$
D_{\mu v}\left(q^{2}\right)=\left(\delta_{\mu v}-\frac{q_{\mu} q_{v}}{q^{2}}\right) D\left(q^{2}\right)
$$

where the expression for $D\left(q^{2}\right)$ obtained by Cornwall is given by

$$
D^{-1}\left(q^{2}\right)=\left[q^{2}+M_{g}^{2}\left(q^{2}\right)\right] b g^{2} \ln \left[\frac{q^{2}+4 M_{g}^{2}}{\Lambda^{2}}\right]
$$

and the coupling constant equal to

$$
\alpha_{s C}\left(q^{2}\right)=\frac{4 \pi}{b \ln \left[\left(q^{2}+4 M_{g}^{2}\left(q^{2}\right)\right) / \Lambda^{2}\right]},
$$

where $M_{g}\left(q^{2}\right)$ is a dynamical gluon mass given by,

$$
M_{g}^{2}\left(q^{2}\right)=m_{g}^{2}\left[\frac{\ln \left(\frac{q^{2}+4 m_{g}^{2}}{\Lambda^{2}}\right)}{\ln \left(\frac{4 m_{g}^{2}}{\Lambda^{2}}\right)}\right]^{-12 / 11}
$$

$\Lambda\left(\equiv \Lambda_{Q C D}\right)$ is the QCD scale parameter.

We will also make use of the running coupling constant obtained by Fischer and Alkofer [27] which is given by

$$
\alpha_{s A}(x)=\frac{\alpha_{A}(0)}{\ln \left(e+a_{1} x^{a_{2}}+b_{1} x^{b_{2}}\right)},
$$

where $x=q^{2}$ and

$$
\alpha_{A}(0)=2.972, \quad a_{1}=5.292 \mathrm{GeV}^{-2 a_{2}} \text {, }
$$




$$
a_{2}=2.324 \text {, }
$$$$
b_{2}=3.169 \text {, }
$$

$$
b_{1}=0.034 \mathrm{GeV}^{-2 b_{2}} \text {, }
$$

and their respective propagator $D\left(q^{2}\right)=Z\left(q^{2}\right) / q^{2}$, where $Z\left(q^{2}\right)$, in Landau gauge, is fitted by

$$
Z(x)=\left(\frac{\alpha_{s A}(x)}{\alpha_{s A}(\mu)}\right)^{1+2 \delta} R^{2}(x),
$$

and

$$
R(x)=\frac{c x^{\mathrm{\kappa}}+d x^{2 \kappa}}{1+c x^{\kappa}+d x^{2 \kappa}}
$$

where the constants appearing in Eq.(11) and Eq.(12) are given by

$$
\begin{aligned}
& \alpha_{A}\left(\mu^{2}\right)=0.9676, \quad \kappa=0.5953, \\
& \delta=-9 / 44, \\
& d=4.6944 \mathrm{GeV}^{-4 \kappa} .
\end{aligned}
$$

All these expressions were obtained from DSE solutions. Eqs.(10) and (11) were obtained in Landau gauge and correspond to a infrared vanishing gluon propagator. The gluon propagator comes with a definite mass scale once the renormalization procedure is defined and the coupling constant fixed at one given scale. The Cornwall result was shown to be gauge invariant, and many of the features of this propagator and coupling constant are discussed by Aguilar and Papavassiliou [28], its mass scale depends on the ratio $m_{g} / \Lambda$.

The details of the elastic differential $p-p$ scattering calculation can be found in Ref.[9], and the comparison of the result with the experimental data of Breakstone et al. at $\sqrt{s}=53$ $\mathrm{GeV}$ [29]is shown in Fig.(1). Note that the agreement between theory and experimental data is good only at small $t$, since at large $t$ values we have contributions from 3-gluon exchange. We see in Fig.(1) that Cornwall's propagator with a dynamical gluon mass of $O(370) \mathrm{MeV}$ fits the data quite well, whereas the Fischer and Alkofer one gives a quite large result for this cross section. We obtained the same result when analysing total hadron-hadron cross section $[9,11]$ and exclusive $\rho$ production in deep inelastic scattering [9].

The integration of Eq.(3) and Eq.(4) picks up an intermediate region of momenta for the gluon propagators, i.e. above the region where the propagator that vanishes at the origin is suppressed and the coupling constant related to it is still large. As we shall see in the next examples most of the problems with this solution comes from the fact that the coupling constant given by Eq.(10) is too large at the origin and up to several hundred $\mathrm{MeV}$, and the integrations that we shall perform are not peaked at a momentum $k^{2}=0$, but are peaked in one region where the product $g^{2}\left(k^{2}\right) D\left(k^{2}\right)$ appearing in Eq.(3) and (4) is not small. As discussed in Ref.[10] there are many indications that the experimental data is better described by a small coupling constant in the infrared.

\section{B. Testing the IR behavior in a perturbative QCD calculation - The $\gamma \rightarrow \pi^{0}$ transition}

Perturbative QCD calculations involve the exchange of gluons at large momenta. These calculations do not provide very

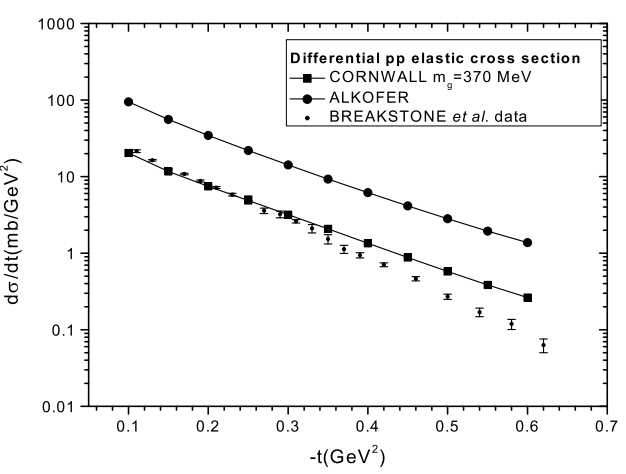

FIG. 1: Differential $p p$ elastic cross section at $\sqrt{(} s)=53 \mathrm{GeV}$ computed within the Landshoff-Nachtmann model for the Pomeron, using different infrared couplings and gluon propagators obtained from DSE solutions.

strong tests for the infrared behavior of the gluon propagator, although a finite infrared gluon propagator gives a natural cutoff for the many infrared divergent parton subprocess cross sections, which, in general, are dependent on the cutoff choice. On the other hand they may be influenced by the behavior of the infrared coupling constant that comes out from DSE solutions, which at intermediate momenta may differ appreciably from the perturbative behavior. It is worth mentioning that several calculations in the literature make use of infrared finite coupling constants. For example, they are natural in the so called Analytic Perturbation Theory (APT) [30] or they appear as an effective charge in several perturbative QCD calculations [31]. In most of the cases these IR finite coupling are fundamental to confront the theoretical and experimental data for several physical quantities. As mentioned before, we shall use the non-perturbative behavior of the coupling constant that comes out from DSE solutions in the sense prescribed by DPT [24].

The photon-to-pion transition form factor $F_{\gamma \pi}\left(Q^{2}\right)$ is measured in single-tagged two-photon $e^{+} e^{-} \rightarrow e^{+} e^{-} \pi^{0}$ reactions. The amplitude for this process has the factorized form

$$
F_{\gamma \pi}\left(Q^{2}\right)=\frac{4}{\sqrt{3}} \int_{0}^{1} d x \phi_{\pi}\left(x, Q^{2}\right) T_{\gamma \pi}^{H}\left(x, Q^{2}\right),
$$

where the hard scattering amplitude $T_{\gamma \pi}^{H}\left(x, Q^{2}\right)$ is given by

$$
T_{\gamma \pi}^{H}\left(Q^{2}\right)=\frac{1}{(1-x) Q^{2}}\left[1+O\left(\alpha_{s}\right)\right]
$$

Using an asymptotic form for the pion distribution amplitude $\phi_{\pi}=\sqrt{3} f_{\pi} x(1-x)$, we obtain [10]

$$
Q^{2} F_{\gamma \pi}\left(Q^{2}\right)=2 f_{\pi}\left(1-\frac{5}{3} \frac{\alpha_{V}\left(Q^{*}\right)}{\pi}\right)
$$


where $Q^{*}=\exp ^{-3 / 2} Q$ is the estimated Brodsky-Lepage-Mackenzie scale for the pion form factor in the scheme discussed in Ref.[32].

In Fig.(2) we compare the photon to pion transition form factor with CLEO data [33]. The curves were computed with different expressions for the infrared behavior of the running coupling constant. We assumed $f_{\pi} \simeq 93 \mathrm{MeV}$ and $\Lambda=300$ $\mathrm{MeV}$. We also made use of a running coupling constant determined by Bloch [34], where the absence of the Landau pole at $q^{2}=\Lambda^{2}$ is reminiscent of APT, which is given by

$$
\begin{aligned}
\alpha_{S B}\left(q^{2}\right)= & \alpha\left(l \Lambda_{Q C D}^{2}\right)= \\
& \frac{1}{c_{0}+l^{2}}\left[c_{0} \alpha_{0}+\frac{4 \pi}{\beta_{0}}\left(\frac{1}{\log (l)}-\frac{1}{l-1}\right) l^{2}\right]
\end{aligned}
$$

where $l=q^{2} / \Lambda_{Q C D}^{2}, c_{0}=15, \alpha_{0}=2.6$, and $\beta_{0}=11-\frac{2}{3} n_{f}$, where $n_{f}$ is the number of flavors. Eq.(16) is also consistent with a propagator that vanishes at $k^{2}=0$. With the coupling constant of Eq.(16) we obtain a fit for the photon-pion transition form factor very far from the experimental data. The result obtained when we use Eq.(10) is not shown and gives an even worse fit. The infrared value of the coupling constant is so large in the case of the coupling constants given by Eqs.(10) and (16), that we are not sure that the perturbative result can be trusted even at such large momentum scale. The momentum scale appearing in Fig.(2) is above the GeV scale, where the DSE solutions for the gluon propagators already assume their perturbative $1 / k^{2}$ behavior. The values of the infrared

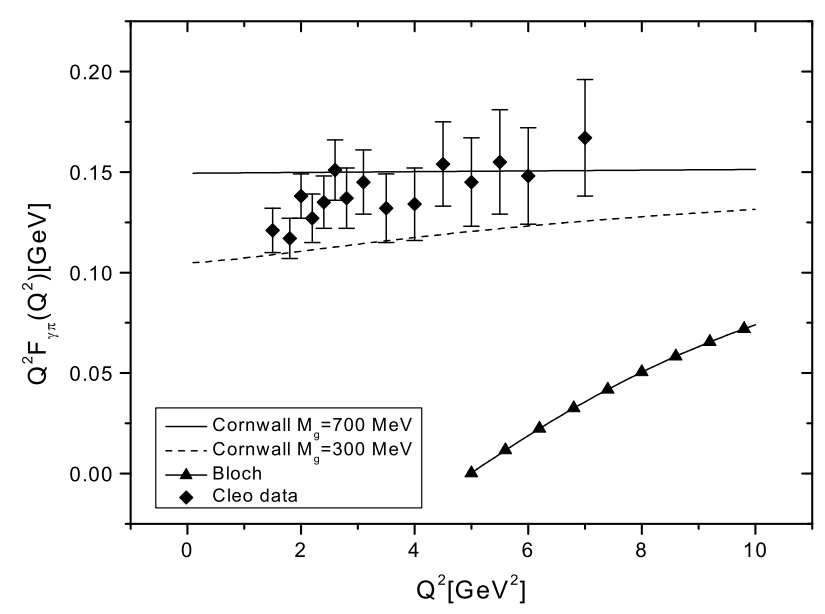

FIG. 2: The $\gamma \rightarrow \pi^{0}$ transition form factor calculated with different expressions for the infrared behavior of the running coupling constant.

coupling constants related to the class of DSE solutions consistent with a vanishing gluon propagator are much stronger than most of the phenomenological estimates of the frozen $\alpha_{s}(0)$ value that we quoted in $\operatorname{Ref} .[10]\left(\alpha_{s}(0) \approx 0.7 \pm 0.3\right)$, and are at the origin of the strange lower curve of Fig.(2). The data is only compatible with Eq.(8), which has a smoother increase towards the infrared region. Perhaps this behavior is actually indicating that the transition to the infrared should be a soft one.

Note that in Fig.(2) the curves obtained with Cornwall's coupling constant do not show large variation in the full range of uncertainty of the dynamical gluon mass. It is interesting that its behavior is quite stable in this case as well as for the pion form factor studied in Ref.[10]. If we had large variations of the infrared coupling constant with the gluon mass scale we could hardly propose any reliable phenomenological test for its freezing value. We stress that the results are obtained for a perturbative scale of momenta and it seems that we have to choose between two possibilities: ( $i)$ This process cannot be predicted by perturbative QCD up to a scale of several $\mathrm{GeV}$, or (ii) some of the DSE solutions are predicting a too large value of the coupling constant in the infrared and the approximations made to determine these solutions are too crude.

We have also computed the perturbative pion form factor $\left(F_{\pi}\left(q^{2}\right)\right)$, and the same happens in that case, i.e. only the solutions for the gluon propagator and coupling constant determined by Cornwall, when plugged into the perturbative QCD expression for $F_{\pi}$, produce the match between the theory and the experimental data. The details of the pion-photon transition form factor calculation can be seen in Ref.[9] and the pion form factor calculation is described in Ref.[10].

\section{Testing the IR behavior in a perturbative QCD calculation - Total hadronic cross sections within the parton model}

Total hadronic and jet cross sections can be calculated in a straightforward way within the parton model (see Ref.[35] for a review); The cross section for producing jets with $p_{T}>p_{T_{\min }}$ through the dominant process $g g \rightarrow g g$ is given by

$$
\begin{aligned}
\sigma_{j e t}(s)= & \int_{p_{T_{\text {min }}^{2}}} d p_{T}^{2} \frac{d \hat{\sigma}_{g g}}{d p_{T}^{2}} \times \\
& \int_{x_{1} x_{2}>4 p_{T}^{2} / s} d x_{1} d x_{2} g\left(x_{1}, Q^{2}\right) g\left(x_{2}, Q^{2}\right),
\end{aligned}
$$

where $g\left(x, Q^{2}\right)$ is the gluon flux and $p_{T_{\min }}^{2}$ is the momentum above which we can use the perturbative calculation of the subprocess differential cross section $d \hat{\sigma}_{g g} / d p_{T}^{2}$. This momentum scale is usually assumed to be larger than $1 \mathrm{GeV}^{2}$. At high energies, the first order perturbative result for the cross section $\hat{\sigma}_{g g}$ can be written in terms of the variable $\hat{s}$ as [36]

$$
\hat{\sigma}_{g g}(\hat{s})=\frac{9 \pi \alpha_{0}^{2}}{m_{0}^{2}} \theta\left(\hat{s}-m_{0}^{2}\right),
$$

where $m_{0}$ is a particle production threshold and $\alpha_{0}$ is an effective value of the running coupling constant. This cross section causes a rapid increase in $\sigma_{j e t}(s)$ if a eikonalization procedure is not used in the calculation. Assuming the simple ansatz for the gluon flux

$$
g(x) \sim \frac{(1-x)^{5}}{x^{J}}
$$


a straightforward calculation yields

$$
\sigma_{j e t}(s) \sim \frac{9 \pi \alpha_{0}^{2}}{m_{0}^{2}}\left(\frac{s}{m_{0}^{2}}\right)^{\varepsilon},
$$

where $\varepsilon \equiv J-1>0$. In this calculation we have neglected some factors in the right hand side of the final expression. Nevertheless, in the limit of large enough $s$ this expression reproduces the expected asymptotic energy dependence of $\sigma_{j e t}(s)$. Moreover, with specific values for $m_{0}$ and $\alpha_{0}$, it is possible to show that at $\sqrt{s} \sim 630 \mathrm{GeV}$, this jet cross section is of order of the $p p$ and $\bar{p} p$ total cross sections [36]. It is also clear that Eq. (20) does violate unitarity.

The simple derivation of the cross section behavior that we have seen above is not too useful due to the following reasons: a) the $m_{0}^{2}$ and the $\alpha_{0}^{2}$ terms in Eq. (20) are totally ad hoc and b) Eq. (20) violates unitarity. Unitarity is recovered with the eikonalization of the model. However the procedure still keeps the ad hoc constants $m_{0}^{2}$ and $\alpha_{0}^{2}$ as parameters that can be obtained only with the data fitting. In Ref. [37] the elementary gluon-gluon cross section was calculated within the dynamical perturbation theory scheme (DPT) [24], where the effective gluon propagator and coupling constant enters into the calculation. With this procedure we eliminate the freedom existent in the previous calculation, due to the choice of $m_{0}^{2}$ and $\alpha_{0}^{2}$, changing $m_{0}^{2}$ by $m_{g}^{2}$ and still have a parameter less in the calculation because the running coupling now is also a function of $m_{g}^{2}$. by

In the eikonal representation the total cross section is given

$$
\sigma_{t o t}(s)=4 \pi \int_{0}^{\infty} d b b\left[1-e^{-\chi_{I}(b, s)} \cos \chi_{R}(b, s)\right]
$$

where $s$ is the square of the total center-of-mass energy, $b$ is the impact parameter, and $\chi(b, s)=\chi_{R}(b, s)+i \chi_{I}(b, s)$ is a complex eikonal function. In the QCD eikonal model with a dynamical gluon mass, henceforth referred to as DGM model, we write the even eikonal as the sum of gluon-gluon, quarkgluon, and quark-quark contributions:

$$
\begin{aligned}
\chi^{+}(b, s) & =\chi_{q q}(b, s)+\chi_{q g}(b, s)+\chi_{g g}(b, s) \\
& =i\left[\sigma_{q q}(s) W\left(b ; \mu_{q q}\right)+\sigma_{q g}(s) W\left(b ; \mu_{q g}\right)\right. \\
& \left.+\sigma_{g g}(s) W\left(b ; \mu_{g g}\right)\right]
\end{aligned}
$$

where $\chi_{p p}^{\bar{p} p}(b, s)=\chi^{+}(b, s) \pm \chi^{-}(b, s)$. Here $W(b ; \mu)$ is the overlap function in the impact parameter space and $\sigma_{i j}(s)$ are the elementary subprocess cross sections of colliding quarks and gluons $(i, j=q, g)$. The overlap function is associated with the Fourier transform of a dipole form factor,

$$
W(b ; \mu)=\frac{\mu^{2}}{96 \pi}(\mu b)^{3} K_{3}(\mu b)
$$

where $K_{3}(x)$ is the modified Bessel function of second kind. The odd eikonal $\chi^{-}(b, s)$, that accounts for the difference between $p p$ and $\bar{p} p$ channels, is parametrized as

$$
\chi^{-}(b, s)=C^{-} \Sigma \frac{m_{g}}{\sqrt{s}} e^{i \pi / 4} W\left(b ; \mu^{-}\right),
$$

where $m_{g}$ is the dynamical gluon mass and the parameters $C^{-}$and $\mu^{-}$are constants to be fitted. The eikonal functions $\chi_{q q}(b, s)$ and $\chi_{q g}(b, s)$, needed to describe the low-energy forward data, are parametrized with terms dictated by the Regge phenomenology:

$$
\begin{gathered}
\chi_{q q}(b, s)=i \Sigma A \frac{m_{g}}{\sqrt{s}} W\left(b ; \mu_{q q}\right), \\
\chi_{q g}(b, s)=i \Sigma\left[A^{\prime}+B^{\prime} \ln \left(\frac{s}{m_{g}^{2}}\right)\right] W\left(b ; \sqrt{\mu_{q q} \mu_{g g}}\right),
\end{gathered}
$$

where $A, A^{\prime}, B^{\prime}, \mu_{q q}$ and $\mu_{g g}$ are fitting parameters.

The innovation in our approach is that the subprocesses cross sections that appear in Eq.(22) are computed within DPT, where the gluon propagator and coupling constants are the ones obtained from DSE solutions. For example, $\sigma_{g g}(s)$ is computed with the help of the Cornwall propagator and coupling constant, where, for simplicity, we neglected the momentum dependence of the running mass in the denominator of the gluon propagator, obtaining

$$
\begin{aligned}
\hat{\sigma}^{D P T}(\hat{s})= & \frac{3 \pi \bar{\alpha}_{s}^{2}}{\hat{s}}\left[\frac{12 \hat{s}^{4}-55 m_{g}^{2} \hat{s}^{3}+12 m_{g}^{4} \hat{s}^{2}+66 m_{g}^{6} \hat{s}-8 m_{g}^{8}}{4 m_{g}^{2} \hat{s}\left[\hat{s}-m_{g}^{2}\right]^{2}}\right. \\
& \left.-3 \ln \left(\frac{\hat{s}-3 m_{g}^{2}}{m_{g}^{2}}\right)\right]
\end{aligned}
$$

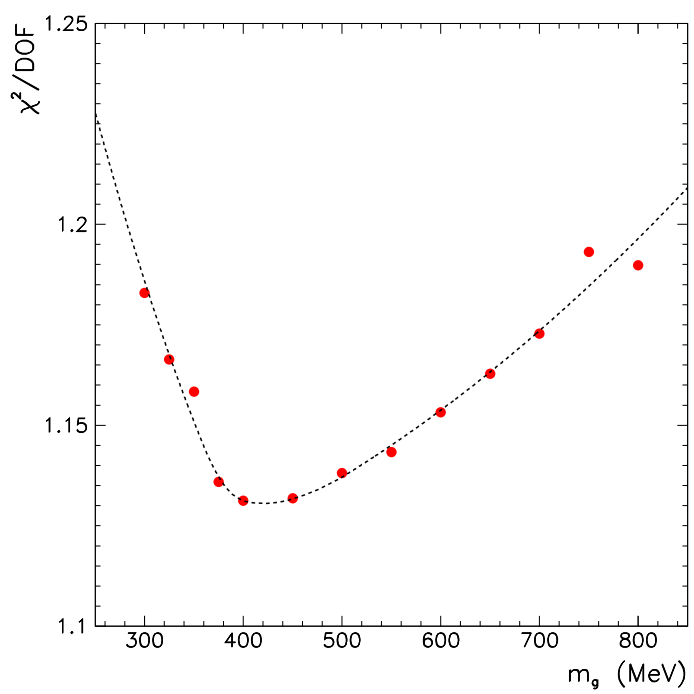

FIG. 3: The $\chi^{2} / D O F$ as a function of dynamical gluon mass $m_{g}$.

Details of this calculation can be found in Ref.[37]. We fitted the $p p$ and $p \bar{p}$ scattering data keeping $m_{g}$ as a free parameter. Our global fit results indicate a minimum value just about $m_{g} \approx 400 \mathrm{MeV}$. These results are shown in Fig.(3), where a general dashed curve is added to guide the eye. Roughly, taking a $5 \%$ variation on the minimal $\chi^{2} / D O F$ value indicated by the general curve, it is possible to estimate a dynamical 
gluon mass $m_{g} \approx 400_{-100}^{+350} \mathrm{MeV}$. This value obtained through data fitting is totally compatible with the ones found by Cornwall and subsequent determinations $[11,17]$. The fits for the total cross sections $\left(\sigma_{t o t}\right)$ can be seen in Fig.(4) in the case of $m_{g}=400 \mathrm{MeV}$.

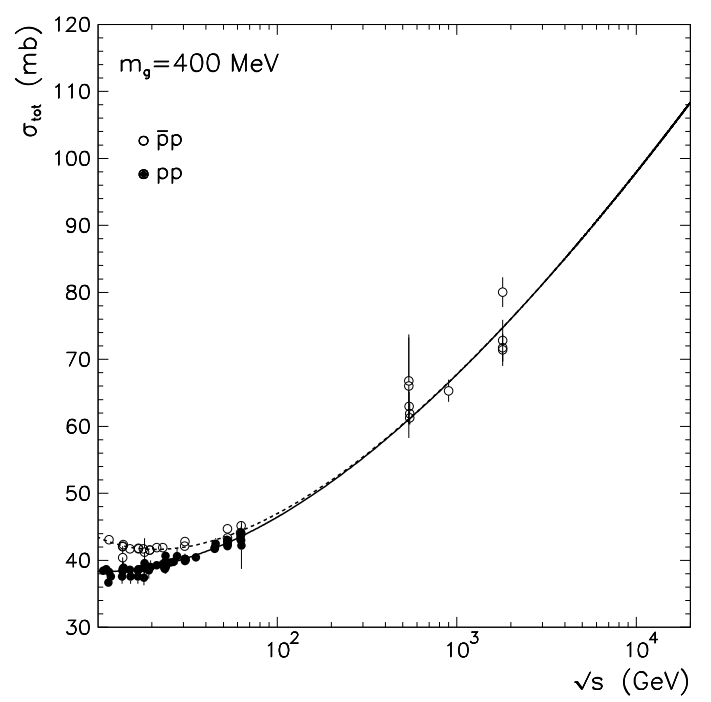

FIG. 4: Total cross section for $p p$ (solid curve) and $\bar{p} p$ (dashed curve) scattering.

It must be noticed that in the cross sections calculations we do have another integration over the gluon propagators and coupling constant expressions weighted by the parton structure functions. In this case we also test the infrared behavior of the gluon propagator because a large part of high energy behavior of the total hadronic cross section is due to soft gluons [35], which means small $\hat{s}$ values in Eq.(27) corresponding to the IR contribution of the gluon propagator. An analysis similar to the $p p$ total cross section calculation shown is this subsection has also been performed in the case of $\gamma-p$ and $\gamma-\gamma$ scattering with the same dynamical gluon mass [38].

\section{CONCLUSIONS}

We discussed DSE solutions for the gluon propagator. These solutions can be divided in two possibilities according to their behavior as $k^{2} \rightarrow 0$, one vanishes at the origin and the other has a value different from zero and consistent with a dynamically generated gluon mass. We should not be surprised with the existence of different solutions because they are originated from different approximations or truncations of the DSE. As one example we could recall that for many years an IR DSE solution for the gluon propagator behaving like $1 / k^{4}$ was very popular, but recent lattice QCD simulations basically discarded such possibility.

Lattice QCD simulations have already proportioned a great improvement in our knowledgement of infrared Green's functions. There are strong indications that the gluon propagator is finite and possibly the same may happens for the coupling constant. This result is important because it may give some information about the confinement mechanism. Unfortunately the lattice result is still not precise enough near the origin of momenta in order to discriminate between the different gluon propagator solutions.

In this work we are advocating that hadronic phenomenology can distinguish between the different IR behaviors of DSE. No matter how we deal with QCD inspired models, like the LN Pomeron model, or just perturbative QCD calculations improved by the use of an effective gluon propagator or coupling constant, we see that Cornwall's propagator is selected by the experimental data. In all the examples discussed in the previous section, we see that the phenomenological information is non-trivial, in the sense that it results from the calculation of physical quantities where the gluon propagator or product of propagators are integrated weighted by different functions (involving different mass scales), and all quantities show agreement with the experimental data for gluon masses that are in the same range of masses predicted by Cornwall several years ago. It is hard to believe that such coincidence is a fortuitous one. These values for the gluon mass lead to a frozen coupling constant at the origin of momenta whose value is not larger than $O(1)$. Certainly an infrared finite coupling constant is welcome for hadronic phenomenology [10, 39]. Maybe future lattice calculations will shed some light on this problem, indicating which DSE approximation is reasonable or not, which confinement scenario is more probable to be at work, and which kind of improved phenomenological calculation can be done with such infrared finite gluon propagator and coupling constant.

\section{Acknowledgments}

I thank A. C. Aguilar and E. G. S. Luna for discussions and collaboration on the topics presented here. I also thank the IRQCD 06 organizers for the hospitality. This research was partially supported by the Conselho Nacional de Desenvolvimento Científico e Tecnológico-CNPq under contract 301002/2004-5.
[1] C. D. Roberts and A. G. Williams, Prog. Part. Nucl. Phys. 33, 477 (1994).

[2] R. Alkofer and L. von Smekal, Phys. Rept. 353, 281 (2001).

[3] C. S. Fischer, J. Phys. G 32, R253 (2006) .

[4] A. C. Aguilar and A. A. Natale, JHEP 0408, 057 (2004).
[5] A. C. Aguilar and A. A. Natale, Int. J. Mod. Phys. A 20, 7613 (2005).

[6] Ph. Boucaud et al., hep-ph/0507104.

[7] V. Sauli, Czech. J. Phys. 55, 1205 (2005).

[8] A. C. Aguilar, A. A. Natale, and P. S. Rodrigues da Silva, Phys. 
Rev. Lett. 86, 152001 (2003).

[9] A. C. Aguilar, A. Mihara, and A. A. Natale, Int. J. Mod. Phys. A 19, 249 (2004).

[10] A. C. Aguilar, A. Mihara, and A. A. Natale, Phys. Rev. D 65, 054011 (2002)

[11] A. Mihara and A. A. Natale, Phys. Lett. B 482, 378 (2000); F. Carvalho, A. A. Natale, and C. M. Zanetti, Mod. Phys. Lett. A, in press, hep-ph/0510172; E. G. S. Luna, Phys. Lett. B 641, 171 (2006); F. Halzen, G. Krein, and A. A. Natale, Phys. Rev. D 47, 295 (1993); M. B. Gay Ducati, F. Halzen and A. A. Natale, Phys. Rev. D 48, 2324 (1993); M. B. Gay Ducati and W. Sauter, Phys. Lett. B 521, 259 (2001); Phys. Rev. D 67, 014014 (2003); hep-ph/0607128; A. I. Soshi, F. D. Steffen, H. G. Dosch, and H. J. Pirner, Nucl. Phys. A 709, 131 (2002); Phys. Rev. D 66, 094019 (2002); Phys. Rev. D 68, 074004 (2003).

[12] A. Cucchieri and T. Mendes, Phys. Rev. D 73, 071502 (2006); Phys. Rev. D 71, 051902 (2005); A. Cucchieri, T. Mendes, and A. Mihara, JHEP 0412, 012 (2004); A. Cucchieri, T. Mendes and A. Taurines, Phys. Rev. D 67, 091502 (2003).

[13] Ph. Boucaud et al., JHEP 0606, 001 (2006); hep-lat/0602006.

[14] E.-M. Ilgenfritz, M. Muller-Preussker, A. Sternbeck, and A. Schiller, hep-lat/0601027; A. Sternbeck, E.-M. Ilgenfritz, M. Muller-Preussker, and A. Schiller, Nucl. Phys. Proc. Suppl. 153, 185 (2006); Phys. Rev. D 73, 014502 (2006); Phys. Rev. D 72, 014507 (2005)

[15] A. Cucchieri, Nucl. Phys. B 508, 353 (1997); Phys. Lett. B 422, 233 (1998); Phys. Rev. D 60, 034508 (1999); H. Suman and K. Schilling, Phys. Lett. B 373, 314 (1996); S. Furui and H. Nakajima, Phys. Rev. D 69, 074505 (2004); J. C. R. Bloch, A. Cucchieri, K. Langfeld, and T. Mendes, Nucl. Phys. B 687, 76 (2004); H. Nakajima and S. Furui, Nucl. Phys. Proc. Suppl. 73, 635 (1999); A. Cucchieri, F. Karsch, and P. Petreczky, Phys. Lett. B 497, 80 (2001); Phys. Rev. D 64, 036001 (2001); O. Oliveira and P. J. Silva, AIP Conf. Proc. 756, 290 (2005); PoS LAT2005, 287 (2005); Phys. Rev. D 74, 034513 (2006).

[16] S. Mandelstam, Phys. Rev. D 20, 3223 (1979).

[17] J. M. Cornwall, Phys. Rev. D 26, 1453 (1982); J. M. Cornwall and J. Papavassiliou, Phys. Rev. D 40, 3474 (1989); J. Papavassiliou and J. M. Cornwall 44, 1285 (1991).

[18] K. Buttner and M. R. Pennington, Phys. Rev. D 52, 5220 (1995); Phys. Lett. B 356, 354 (1995).

[19] V. N. Gribov, Nucl. Phys. B 139, 1 (1978).

[20] D. Zwanziger, Nucl. Phys. B 412, 657 (1994).

[21] R. F. Sobreiro and S. P. Sorella, hep-th/0504095, lectures given at 13th J. A. Swieca Summer School on Particle and Fields, Campos do Jordão, Brazil, 2005; S. P. Sorella, Ann. Phys. (NY)
321, 1747 (2006); R. F. Sobreiro, S. P. Sorella, D. Dudal and H. Verschelde, Phys. Lett. B 590, 265 (2004); D. Dudal, R. F. Sobreiro, S. P. Sorella, and H. Verschelde, Phys. Rev. D 72, 014016 (2005); D. Dudal et al., JHEP 0401, 044 (2004); A. R. Fazio, V. E. R. Lemes, M. S. Sarandy, and S. P. Sorella, Phys. Rev. D 64, 085003 (2001); M. A. Capri et al., Phys. Rev. D 72, 085021 (2005); J. A. Gracey, JHEP 0605, 052 (2006); M. A. L. Capri et al., hep-th/0603167.

[22] R. Alkofer, C. S. Fischer, and F. J. Llanes-Estrada, hep$\mathrm{ph} / 0607293$.

[23] P. V. Landshoff and O. Nachtmann, Z. Phys. C 35, 405 (1987).

[24] H. Pagels and S. Stokar, Phys. Rev. D 20, 2947 (1979).

[25] Ya. Ya. Balitsky and L. N. Lipatov, Sov. J. Nucl. Phys. 28, 822 (1977); E. A. Kuraev, L. N. Lipatov, and V. Fadin, Soviet Physics JETP 45, 199 (1977).

[26] H. Chehime et al., Phys. Lett. B 286, 397 (1992).

[27] C. S. Fischer, R. Alkofer, and H. Reinhardt, Phys. Rev. D 65, 094008 (2002); C. S. Fischer and R. Alkofer, Phys. Lett. B 536, 177 (2002); R. Alkofer, C. S. Fischer, and L. von Smekal, Acta Phys. Slov. 52, 191 (2002).

[28] A. C. Aguilar and J. Papavassiliou, hep-ph/0610040, to appear in JHEP; talk at IRQCD 06, june/2006, Rio de Janeiro, Brazil, hep-ph/0610150.

[29] A. Breakstone et al., Nucl. Phys. B 248, 253 (1984).

[30] D. V. Shirkov and I. L. Solovtsov, Phys. Rev. Lett. 79, 1209 (1997); A. V. Nesterenko and J. Papavassiliou, Int. J. Mod. Phys. A 20, 4622 (2005); Phys. Rev. D 71, 016009 (2005); A. C. Aguilar, A. V. Nesterenko, and J. Papavassiliou, J. Phys. G 31, 997 (2005).

[31] S. J. Brodsky, E. Gardi, G. Grunberg, and J. Rathsman, Phys. Rev. D 63, 094017 (2001); S. J. Brodsky, S. Menke, C. Merino, and J. Rathsman, Phys. Rev. D 67, 055008 (2003).

[32] S. J. Brodsky, C. R. Ji, A. Pang, and D. G. Robertson, Phys. Rev. D 57, 245 (1998).

[33] J. Gronberg et al., (CLEO Collaboration), Phys. Rev. D 57, 33 (1998).

[34] J. C. R. Bloch, Phys. Rev. D 66, 034032 (2002).

[35] M. M. Block et al., Nucl. Phys. B (Proc. Suppl.) 12, 238 (1990).

[36] B. Margolis et al., Phys. Lett. B 213, 221 (1988); T. K. Gaisser and F. Halzen, Phys. Rev. Lett. 54, 1754 (1985); G. Pancheri and Y. Srivastava, Phys. Lett. B 159, 679 (1985).

[37] E. G. S. Luna et al., Phys. Rev. D 72, 034019 (2005).

[38] E. G. S. Luna and A. A. Natale, Phys. Rev. D 73, 074019 (2006).

[39] G. Prosperi, M. Raciti and C. Simolo, hep-ph/0607209. 